\title{
श्री कृष्ण से संबंधित बंदिशों का आध्यात्मिक महत्व
}

ज्योति शर्मा

शोधार्थी, संगीत - विभाग, पंजाब विश्वविद्यालय, चण्डीगढ़

भगवान श्री कृष्ण का कथन है - वेदानां सामवेदोऽस्मि अर्थात वेदों में मैं सामवेद हूँ। वेदों का कथन है - अगुष्ठमात्र: पुरूषोऽन्तरात्मा सदा जनानां हदय सन्निविष्ठ:। नाद ही ब्रह्मा है। व्यापक परमात्मा में ही आत्मा अनुरंजन करती है। 'नादाधीनं जगत् सर्वम्' स्वयं न्यायेश्वर भगवान नाद के अधीन है। भगवान के हाथ में वेणु है और उन्होंने नारद से कहा है कि जहाँ-जहाँ मेरे भक्तगण गायन करते है वहाँ- वहाँ मैं उपस्थित रहता हूँ। श्री कृष्ण जी नारद से कहते है -

नाहं वसाभि बैकुण्ठे योगिनां हृदय न च ।

मद्भक्ता यत्र गायन्त्रि तत्र तिष्ठामि नारद:

अर्थात् न तो मैं बैकुण्ठ में निवास करता हूँ और न योगियों के हृदय में। हे नारद मेरे भक्त जहाँ गान करते है मैं वही स्थित होता हूँ। कृष्ण का कोई भी रूप हो वे सदा सर्वदा आराध्य है। पूजा की कोई भी क्रिया संगीत विहीन नहीं होती। भक्त तो सिर्फ प्रभु की दृष्टि का याचक होता है। भगवान श्री कृष्ण के सभी रूपों का विवरण जैसे बाल कृष्ण, गोपी बिहारी श्री कृष्ण संबंधी जो गीत है उनका भारतीय साहित्य और संगीत पर बहुत प्रभाव पड़ा है।

वैष्णवों का कथन है कि संगीत श्री कृष्ण की ही देन है। साधारणत: संगीत का गुण तन्मयता है और इसके ऊर्ध्वमुखी होने पर मनुष्य ईश्वर की ओर प्रवृत होता है। भगवान श्री कृष्ण का संबंध संगीत से प्रत्येक काल में रहा है।

कृष्ण वैदिक देवता विष्णु के अवतार माने जाते है। पुराण साहित्य का तो सर्वाधिक चर्चित एवं प्रिय अवतार श्री कृष्ण ही है। भगवतपुराण में ता श्री कृष्ण को पूर्णावतार भगवान माना गया है तथा अन्य अवतारों को अंशावतारा कहा है। वैदिक काल के पश्चात त्रिदेव वाद का विकास हुआ जिसमें ब्रह्मा, विष्णु तथा शिव आराध्य देव हुए। वैदिक सक्तों में वर्णित रूप के आधार पर इन देवताओं के मानवीकृत रूप बने। विष्णु का रूप बहुत सुंदर था तथा गुण जगत् का पालन करना। इसलिये विष्णु की उपासना अधिक प्रचलित हुई। पौराणिक काल में विष्णु के विभिन्न रूपों की आराधना स्तोत्रगान द्वारा हुई। विष्णु के अवतार राम और कृष्ण हुए। विष्णु के अवतारों में राम और कृष्ण की स्थिति अन्य अवतारों से भिन्न है। राम को तुलसी ने मर्यादा पुरूषोत्तम भगवान के रूप में चित्रित किया तो कृष्ण को सर्वथा भुलाया नहीं। वस्तुतः मध्ययुगीन भक्ति का मेरूदण्ड राम और कृष्ण की भक्ति ही है और इन्ही दो आराध्य इष्टदेवों के विम्रह विविध रूप में पूजा-अर्चा के स्वीकृत हुए। विष्णु के दस अवतार माने गये है और इनकी स्वतंत्र पुराणों में व्याख्या की गयी। कालान्तर में राम और कृष्ण आराध्य देव हुए।

गीता के ये श्लोक भी इस तथ्य की ओर संकेत करते है:- 
यदा यदा हि धर्मस्य गलानि भावति भारत।

अभ्युत्यानमधर्मस्य तदात्मान सृजाम्यह्म।

परित्राणाय साधनां विनाशाय च दृष्कृताम

धर्म संस्थापनार्थाय सभवानि युगे युगे।

श्री कृष्ण जो कि सोलह कला सम्पूर्ण माने जाते है। समस्त भारत में उपास्य देव प्रतिष्ठित हुए। इस समय स्तुतिगान स्तोत्र के रूप में रचा गया। प्राचीन काल में ही स्तोत्रगान के साथ - साथ नामों का गुणगान भी प्रचलित हुआ।

मध्यकाल को भक्ति काल माना गया है। सगुण भक्ति धारा में राम और कृष्ण का गुणगान हुआ। तुलसीदास जी ने 'रामचरितमानस' नामक अमर गेय काव्य की रचना तो सूर, मीरा आदि अष्टछाप के कवियों ने कृष्ण की स्तुति में विभिन्न काव्य रचना कर गान किया। इन भक्त कवियों ने घूम घूम कर गायन द्वारा कृष्ण परक साहित्य का प्रचार किया। इसका प्रभाव शास्त्रीय संगीत की रचनाओं पर भी पड़ा। सूर जैसा भाव मीरा जैसा प्रेम और तुलसी जैसी श्रद्धा रख कर भक्ति तथा अध्यात्मिक संगीत प्रस्तुत जाए तो मनुष्य का जीवन सफल हो जाए। मुरलीधर श्री कृष्ण से संबंधित आधुनिक काल तक विभिन्न कवि या गीतकार लिखते चले आए।

संगीत ने कृष्ण परम्परा का प्रवर्तन 2 वीं शताब्दी के पूर्व ही सम्पन्न हो चुका था। हरिवंश के अनुसार श्री कृष्ण की सभा में गंधर्व, अपसरा, सूत आदि कलाकारों को स्थान प्राप्त था। संगीत म जब पृथक से कृष्ण की परम्परा विद्यमान हो तो कृष्ण भक्त कवियों ने अपने अपने ढंग से इस परम्परा का निर्वाह एक पल्लवन किया है।

श्री कृष्ण से संबंधित संगीतिक रचनाएं एवं शैलियाँ जैसे - सामगान, गाथागान, ध्रवा, जाति, प्रबंध, धुपद एवं ख्याल की पद, गीत प्रचार में है। पष्टिमार्गीय हवेली संगीत की परम्परा महाप्रभु के पुत्र विट्ठल दास जी के समय से गणना में आती है। महाप्रभु जी आध- प्रवर्तक है। श्री कृष्ण मेरे सर्वस्व है, मेरे शरण है...... इसी भावना का पोषक यह संप्रदाय अपने कीर्तनों के लिए जाना जाता है। वैष्णव धर्म भी परंपरागत कीर्तनों के लिए प्रसिद्ध है। बंगाल के चैतन्य संप्रदाय के कीर्तनों की भाषा बंगला है। कर्नाटक संगीत भी मुख्यतः कीर्तन संगीत ही है। छोटे कीर्तन को 'कृति' और जिसके साथ नृत्य हो उस कीर्तन को 'पद' कहा जाता है। इन पदो का मुख्य साहित्य वैष्णव सम्प्रदाय के अन्तर्गत है। गायन शैली धीमी और भावप्राण है। इनकी भाषा का आधार तमिल तेलगू है। यह ध्रुपद धमार अर्वाचीन उत्तर भारतीय संगीत के स्तंभ है। यह भावपूर्ण मंद के गति के कीर्तन गाए जाते हैं और इनका विषय भी राधाकृष्ण की भक्ति ही है। ख्याल गायन शैली का प्रचार ध्रुपद धमार गायन शैली के उपरान्त हुआ। ख्याल गायक भी प्राय: मुरलीधर श्री कृष्ण से संबंधित ख्याल की बदिश जब गाते है तो उस में मुरली वाद्य का विवरण अवश्य प्राप्त होता है। 
कीर्तन देव गोविन्द : अर्थात् गायन द्वारा ही भगवप्राप्ति होती है और वही परमशक्ति का आधार है। कृष्ण ही संगीत है और संगीत ही इसी आध्यात्मिक महत्ता के कारण कृष्ण भक्तों ने भजन व कीर्तन द्वारा इष्ट को रिझाने का सफल प्रयत्न किया था। भागवत में संगीत की आध्यात्मिक महत्ता का वर्णन मिलता है -

दोषनिधि कलियुग में महान गुण है कि भगवान कृष्ण के कीर्तन से मनुष्य लौकिक आसक्ति से छूट जाता है-

\section{कीर्तनादेव कृष्णस्य मुक्त संग: परंब्रजते।}

मनुष्य एक चिन्तनशील प्राणी है। चिरकाल से वह उस प्रेरक-स्त्रोत की चिरूतर खोज में भटकता रहा है, जहां से उसे सतत अग्रसर होने की शक्ति प्राप्त होती है। प्रेम भाग ने उसकी उत्कृष्ट अभिलाषा की पूर्ति की है। विश्व के साहित्य एवं कलाओं में अन्य भावों व सम्वेदनाओं की उपेक्षा प्रेमभाव का व्यापक चित्रण प्रचुरता से मिलता है। यदि प्रेम लौकिक हुआ तो मानव ने इस लौकिक आलौकिक कार्य कर दिखाये और यदि वह ईश्वरोन्मुख हुआ तब अध्यात्म क्षेत्र का वह दिव्य स्वरूप दूसरों के लिए भा सुलभ हो सका।

अध्यात्मिकता भारतीय संगीत की नहीं, अपितु सम्पूर्ण भारत की आत्मा का आलैकिक श्रृंगार है। आध्यात्मिकता की यह परम्परा कृष्ण भक्तों के काव्य में सांगोपांग वर्णित मिलती है। विशेषत: पुष्टिमार्गीय वैष्णव - देवालय - संगीत - भारतीय - संगीत - जगत् में प्राचीनतम पुष्ट व प्रशंसनीय परम्परा है। यदि कहा जाये कि इस परम्परा में संगीत का अध्यात्म अवरित हुआ तो अतिशयोक्ति नहीं।

कृष्ण से संबंधित बंदिशों का विषय के अन्तर्गत लीला, होरी, प्रेम, रास, श्रृंगार, दर्शन, अध्यात्म और कर्मयोग में भगवान श्री कृष्ण की नाना प्रकार की लीलाएँ, होरी गीत में बंधे हुए धमार, दीपचंदी इत्यादि तालों रचितत मिलते है जिस का विषय प्रेम, होरी संबंधी गीतों में होता है। वेणु गीत, गोपिका गीत, युगल गीत, भ्रमर गीत, रास इत्यादि कृष्ण संबंधी बंदिशों का वर्णन मिलता है जो कि भावात्मक और अनुकूल वातावरण का दृष्टिगोचर होता है। ख्याल की इन परम्परागत बंदिशों में नियमों का पालन किया जाता है जिस में परम संतोष की प्राप्ति होती है।

अष्टछाप व अन्य कवियों ने अपने काव्य के माध्यम से तथा ख्याल रचनाओं के वाग्ययेकारों व गायकों ने कृष्ण का गुणगान संगीत के माध्यम से खूब प्रचार-प्रसार हुआ। ख्याल के स हित्य में भक्ति अध्यात्मिक, श्रृंगार, लौकिक इत्यादि बंदिशे रची गई जिन्हें विभिन्न वाग्ययेकारों ने अपनी साधना से परिष्कृत कर समाज में प्रस्तुत किया। अनेकोमं बंदिशे गायकों द्वारा गायी गई जो अध्यात्मक की भावना दर्शाता है। 


\section{राग भीमपलासी}

स्थाई

भजमन निसदिन श्याम सुंदर।

सुख के सागर श्री राधावर।।

अंतरा

सकल जगत के पालन करता।

राग वृंदावनी सारंग

करत कृपा नितनिज भक्तन पर।।

स्थाई

वन वन ढूंढन जाऊँ।

कित हूं छिप गये कृष्ण मुरारी।।

अंतरा

शीश मुकुट और कानन कुण्डल।

बंसाधर मन रंग फिरत गिरधारी।।

राग रवमाज

स्थाई

बलि बलि जाऊँ मुखारबिंदु के।

कान्ह कुंवर गिरधारी हे प्रभू।।

अंतरा

जैसी शोभा चंद्रवदन की ।

उपमा को न तुम्हारी हे प्रभू॥

देश- देशांतर-व्यापी मानव कल्याण हेतु भक्तिभावना की अनुभूति का प्रतिफल होने के परिणामस्वरूप, कृष्ण भक्तिधारा के निबंध-प्रवाह में संगीत अपनी आध्यात्मिक प्रवृत्ति तथा विश्वव्यापी महत्ता के कारण प्रमुख माध्यम आधार व उपादान बना। संगीत की रमणीयता के कारण ही कृष्ण भक्ति ने भी चिन्तवृति के लिए अन्य साधनों के साथ संगीत को भी अपनाया है।

\section{सन्दर्भ ग्रन्थ सूचि}

गुप्ता उषा, हिन्दी कृष्ण भक्तिकालीन साहित्य में संगीत, प्रकाशक लखनऊ विश्वविद्यालय, प्रभ माव्रति, 2006 नगेन्द्र, रस सिद्धांत, नेशनल पब्लिशिंग हाऊस दिल्ली, 1974

श्री मदभगवद्गीता, गीताप्रेस, गोरखपुर

श्री मद्भागवतमहापुराण, महर्षि वेदव्यास, गीताप्रेस, गोरखपुर

शर्मा डॉ. सुनीता, भारतीय संगीत का इतिहास, संजय प्रकाशन दिल्ली प्र. सं. 1996

गैशेला वाचस्पति, भारतीय संस्कृति और कला, हिन्दी ग्रंथ अकादमी, लखनऊ 1973

शर्मा पंकज माला, सामगान: उद्भव व्यवहार एवं सिद्धांत, कान्यायन वैदिक साहित्य प्रकाशन होशियारपुर, 1996

शास्त्री देवऋणि, कलानाथ संस्कृत साहित्य का इतिहास, धामाणी मार्किट की गलि, चौड़ा रास्ता, जयपुर संस्करण 2009

विष्णु पुराण, गीता प्रेस गोरखपुर।

पं विष्णु - नारायण भातखण्डे, क्रमिक पुस्तक मालिका भाग - 3, 1994 